\title{
MANEJO DE ARVENSES
}

Luis Fernando Salazar Gutiérrez * - Fabio Alexis Torres Angarita **

El Manejo Integrado de Arvenses (MIA) se basa en entender la importancia de la cobertura del suelo para prevenir su erosión.

Durante los primeros 2 años de edad del cultivo, el café es muy sensible a la competencia de las arvenses (plantas acompañantes de los cultivos), y el suelo se encuentra más propenso a la erosión por efecto de las lluvias. Igualmente, durante la etapa productiva las arvenses más agresivas compiten fuertemente por el agua y los nutrientes del suelo, llegando a reducir la producción en más del 60\%.

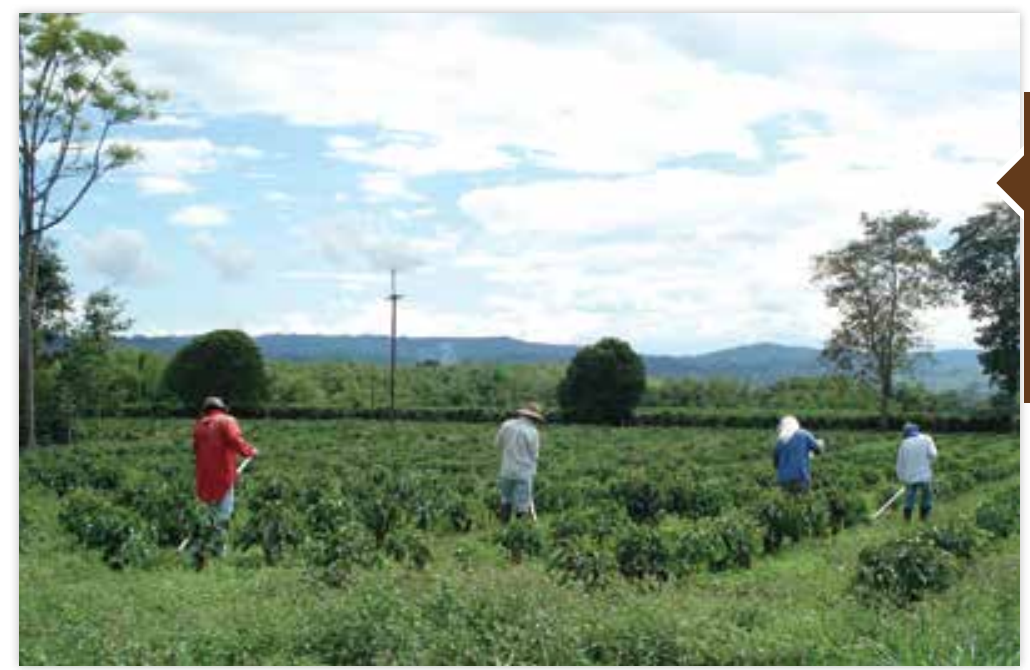

Personas realizando el Manejo Integrado de Arvenses en el cultivo del café.

La cobertura del suelo por las arvenses nobles permite que el agua se almacene o retenga, y esté fácilmente disponible en la zona de raíces del cultivo, las arvenses también protegen al suelo del impacto del golpe de las gotas de lluvia, con lo cual se disminuye la erosión entre el $50 \%$ al $97 \%$. 
Para implementar el MIA en la finca cafetera se deben integrar los diferentes métodos de control: el químico (con herbicidas), el manual, el mecánico (cortes con machete o guadañadora) y el cultural que se refiere a las buenas prácticas del cultivo (densidad de siembra, variedades mejoradas y coberturas con residuos del cultivo, entre otros); lo anterior para hacer controles selectivos de arvenses o en parcheos sobre las arvenses más agresivas y dar lugar a aquellas benéficas o conocidas como nobles.
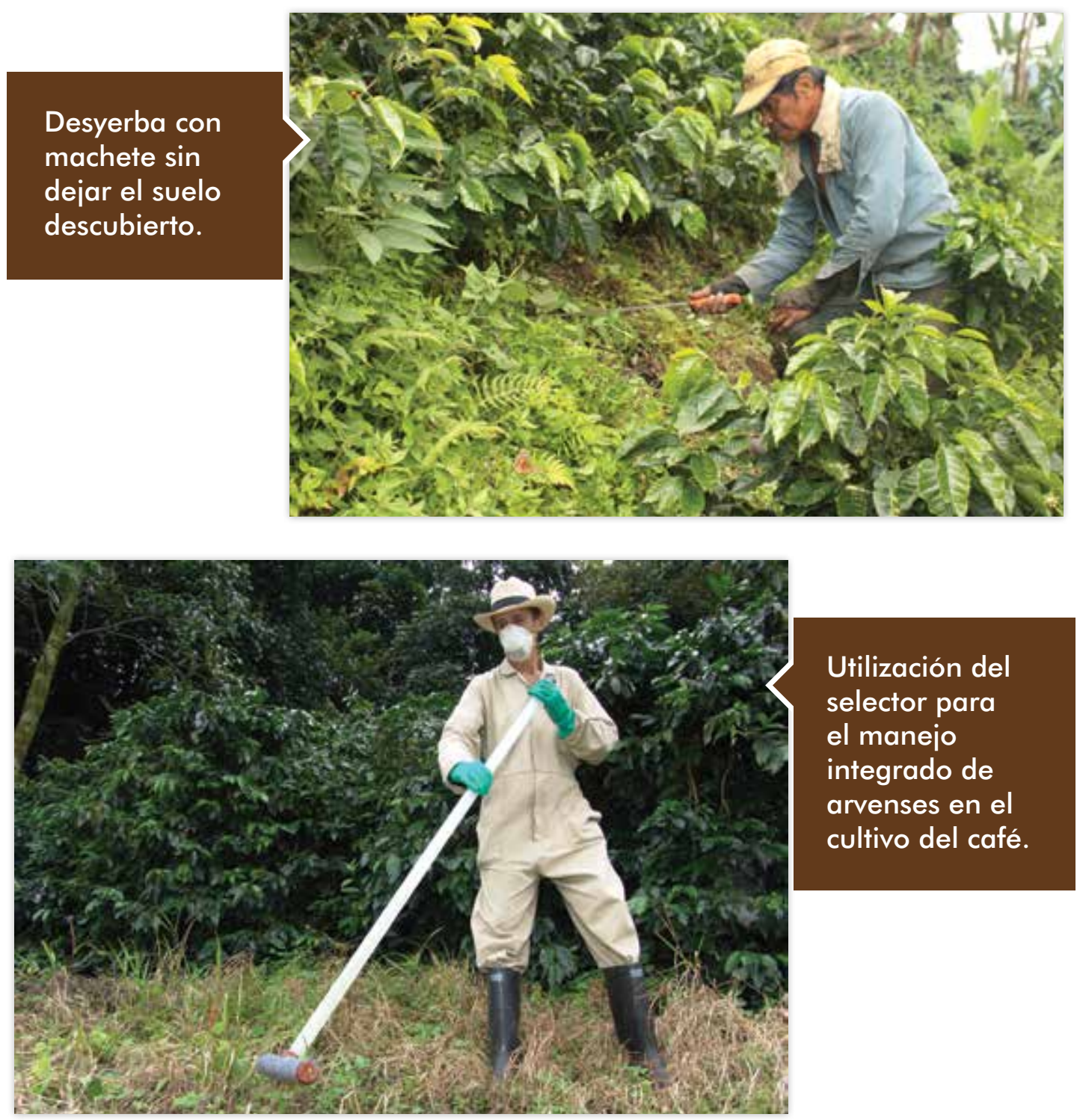

Utilización del selector para el manejo integrado de arvenses en el cultivo del café. 
La implementación de un solo método de control presentará dificultades debido a que las arvenses, como seres vivos, con el tiempo buscan formas de escapar a las distintas medidas de control, por ejemplo, en la zona cafetera se ha dado el caso de aparición de arvenses resistentes a los herbicidas. Igualmente, el uso de un único método de control eleva los costos de producción. Por ello, deben integrarse los diferentes métodos de manejo asegurándose de no desnudar el suelo.

Una forma de ser más rentable en la actividad cafetera es con la adopción del MIA, éste apunta a mejorar la rentabilidad en cuatro formas:

1. Reducción de los costos de las desyerbas. En las fincas cafeteras este sistema puede disminuir los costos entre el $20 \%$ para el primer año y $45 \%$ en el segundo año de establecimiento del café, por la reducción en la mano de obra e insumos necesarios para su realización.

2. Mantenimiento $o$ aumento de la productividad debido a controles más oportunos de arvenses. Las coberturas nobles protegen el suelo de la erosión sin afectar la producción del cultivo.

3. Menor riesgo de aparición de arvenses resistentes a los herbicidas. Cuando las arvenses se vuelven resistentes a los herbicidas siguen compitiendo con el cultivo y se pierde la inversión y el esfuerzo hecho para su manejo.

4. Prevención de la erosión del suelo. Cuando el suelo llega a un estado de erosión avanzado (pérdida del horizonte orgánico) los rendimientos pueden disminuir hasta el $51 \%$, aun con la aplicación de fertilizantes. De allí la importancia de proteger los suelos con coberturas nobles. 
Por lo anterior, es importante recordar la finalidad del MIA que consiste en mantener el suelo con coberturas para conservarlo al igual que al agua, sin que se afecte la productividad y los costos de producción, lo cual se logra al disminuir la proporción de las poblaciones de arvenses agresivas y favorecer el establecimiento de aquellas de fácil manejo o coberturas nobles.

Aspecto de un cultivo de café productivo con adecuado manejo integrado de arvenses.

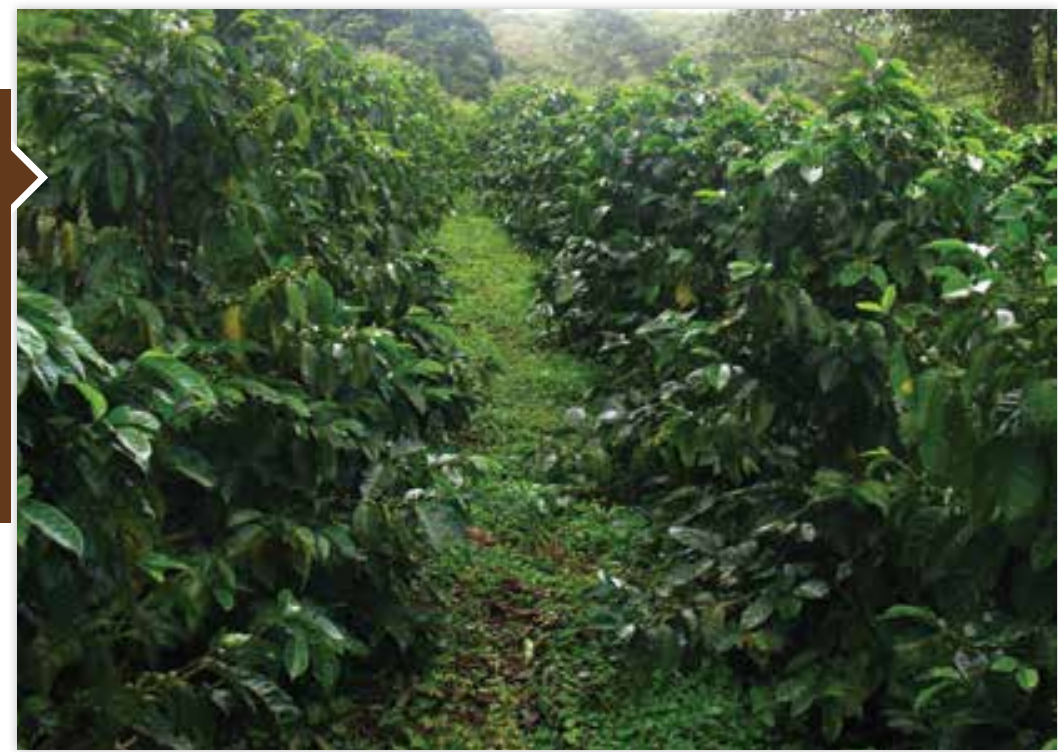

Las arvenses nobles son plantas de porte bajo, crecimiento rastrero, con raíz superficial, con cubrimiento denso del suelo y no interfieren con el desarrollo y producción de la planta de café, si no están presentes en la zona de raíces (plato).

Las arvenses agresivas pueden presentar una o varias de las siguientes características: alta producción de semillas, raíces profundas y abundantes, son de difícil control manual, mecánico o químico, con estructura semileñosa y hábito trepador. Si tiene dudas sobre el tipo de arvenses en su finca consulte con el Servicio de Extensión. 

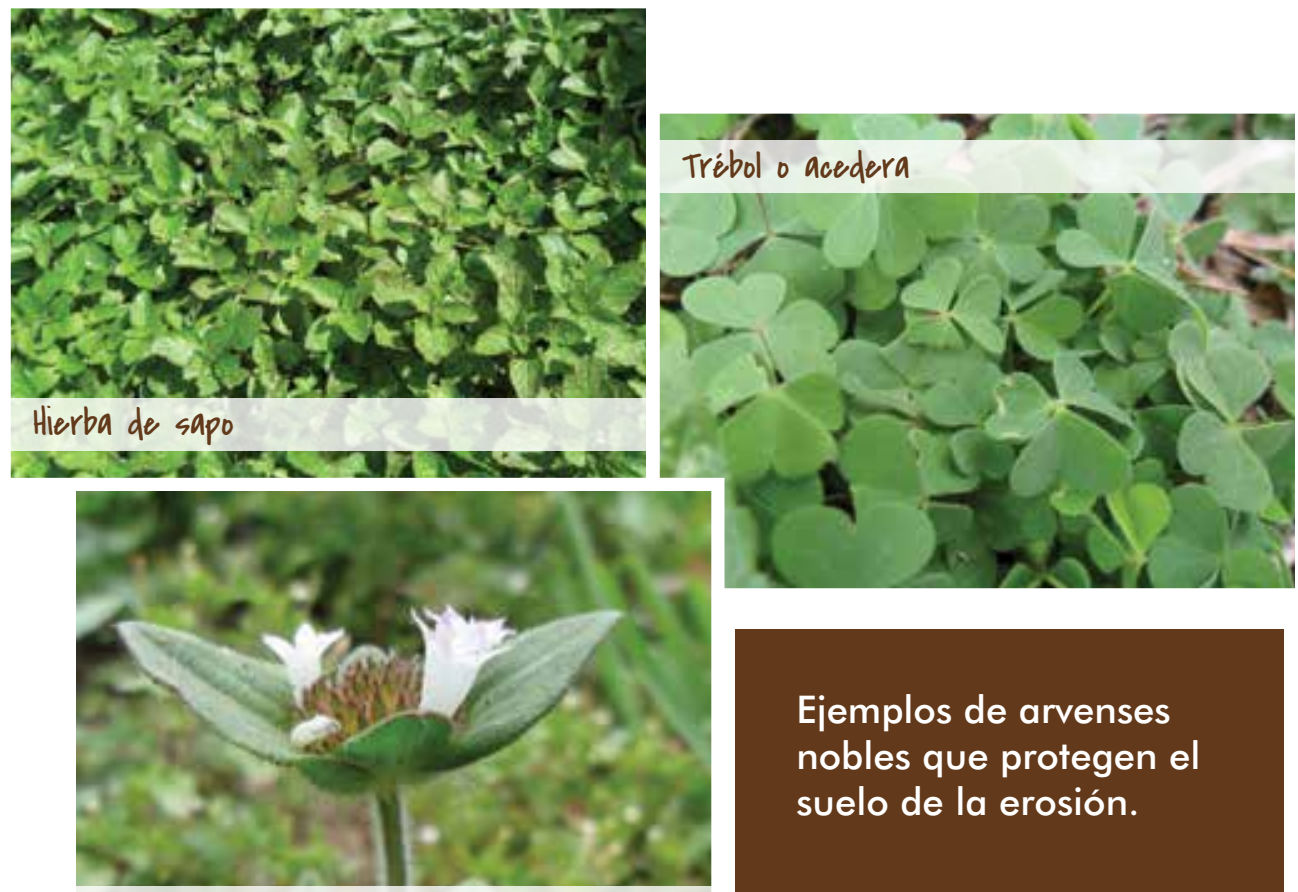

Botoncillo

Ejemplos de arvenses nobles que protegen el suelo de la erosión.
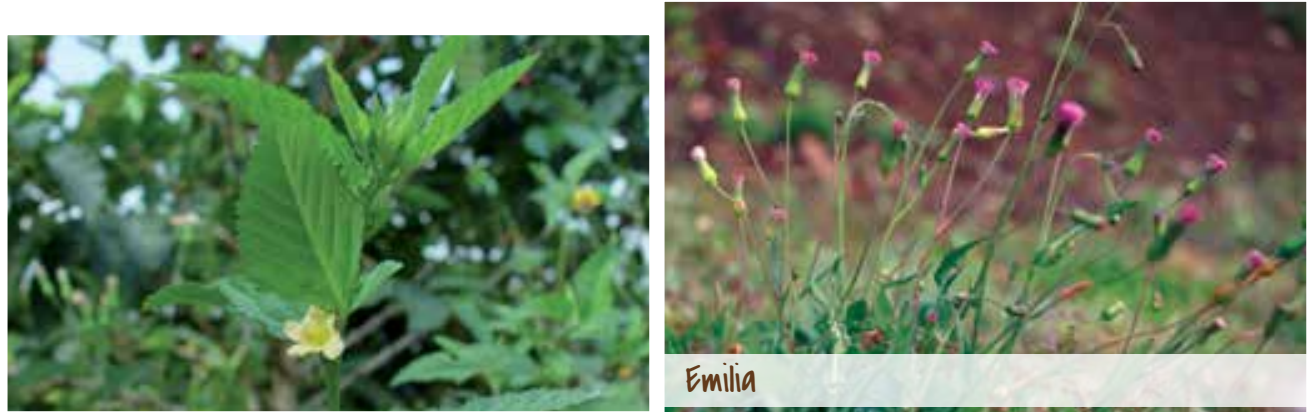

Escobadura

Emilia
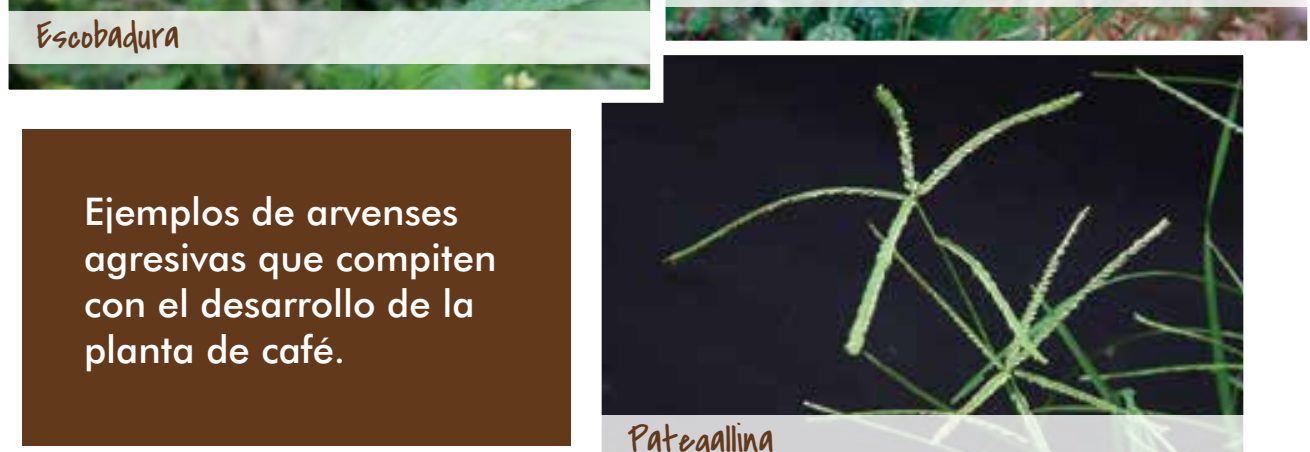

Pategallina 


\section{Recomendaciones para una exitosa implementación del MiA}

1. El primer paso es recibir capacitación sobre el tema y llevar estos conocimientos a las personas de la empresa cafetera involucradas con el manejo del cultivo.

2. Reconozca las arvenses por su grado de competencia. Para lo anterior puede elaborar un listado con las arvenses más agresivas y las nobles en la finca, cuenca o vereda.

3. Lleve el registro de los costos en los cuales incurre al realizar el manejo de arvenses. Registre los costos de las desyerbas en distintos lotes de la finca y compárelos frente al manejo tradicional. Si los costos del MIA en su finca son más altos, consulte con el Servicio de Extensión.

4. Utilice en forma adecuada las herramientas para el MIA como son el selector de arvenses, los equipos de aspersión, machete y guadañadora, y realice el mantenimiento de los mismos. Utilice la dosis correcta del herbicida, atendiendo las recomendaciones del Servicio de Extensión.

5. Implemente el MIA en los diferentes sistemas de producción de café, así:

- Si las arvenses presentan una altura mayor de $20 \mathrm{~cm}$ o están en estado de floración o producción de semillas haga un corte con guadañadora o machete a una altura de 3 a $5 \mathrm{~cm}$ del suelo.

- Después de 15 a 20 días, antes de que las arvenses comiencen su floración, haga parcheos selectivos con herbicida sobre las arvenses más agresivas, preferiblemente con el selector de arvenses o con un equipo de aspersión con pantalla protectora.

- Continúe realizando los parcheos periódicamente, evitando que las arvenses alcancen su estado de floración o producción de semillas. Si las arvenses alcanzan esta etapa repita el corte con machete o guadaña, sin desnudar el suelo. Esto le permitirá disminuir la presencia de arvenses agresivas y mejorar el cubrimiento dado por las arvenses nobles o de fácil manejo. 
Planilla para el registro de costos del manejo integrado de arvenses en la empresa cafetera.

\begin{tabular}{|l|l|l|l|l|l|}
\hline Edad del cultivo & $0-1$ año & $1-2$ años & $2-3$ años & $3-4$ años & 4 y más años \\
\hline Nombre del lote & & & & & \\
\hline Área de lote $\left(m^{2}\right)$ & & & & \\
\hline Labor* & & & & \\
\hline Duración de la labor & & & & \\
\hline Jornal/ha & & & & \\
\hline Insumo (L/ha) & & & & \\
\hline $\begin{array}{l}\text { Costo mano de } \\
\text { obra por hectárea }\end{array}$ & & & & \\
\hline $\begin{array}{l}\text { Costo insumos por } \\
\text { hectárea** }\end{array}$ & & & & \\
\hline Costo total
\end{tabular}

* Labor: plateo manual, plateo con herbicida, corte con guadañadora o machete, parcheo con fumigadora, parcheo con selector, control manual.

** Costo de los insumos/ha: (por ejemplo: herbicida, lubricantes, combustibles).

6. Esté atento a la aparición de nuevas especies de arvenses agresivas en su cultivo o a la presencia de arvenses resistentes a los herbicidas; de encontrarlas consulte con su Extensionista.

7. En épocas secas realice un plateo manual en las plantas de café en estado de levante, utilice el material vegetal que resulte de esta labor como cobertura muerta en los platos de la planta, para conservar la humedad del suelo y retrasar el crecimiento de las arvenses en esta zona. 
En las calles controle con machete o guadañadora las arvenses agresivas las cuales compiten por agua y nutrientes, y permita el establecimiento de arvenses nobles o de baja competencia.

8. Realice el plateo o control de arvenses en la zona de raíces, en forma manual y utilice el herbicida de tipo pre-emergente, con el cual puede llegar a tener el plato libre de arvenses por más tiempo. Recuerde que antes de la aplicación de este tipo de herbicida, el plato debe estar libre de arvenses y disponer de buena humedad en el suelo. Consulte con su Extensionista.

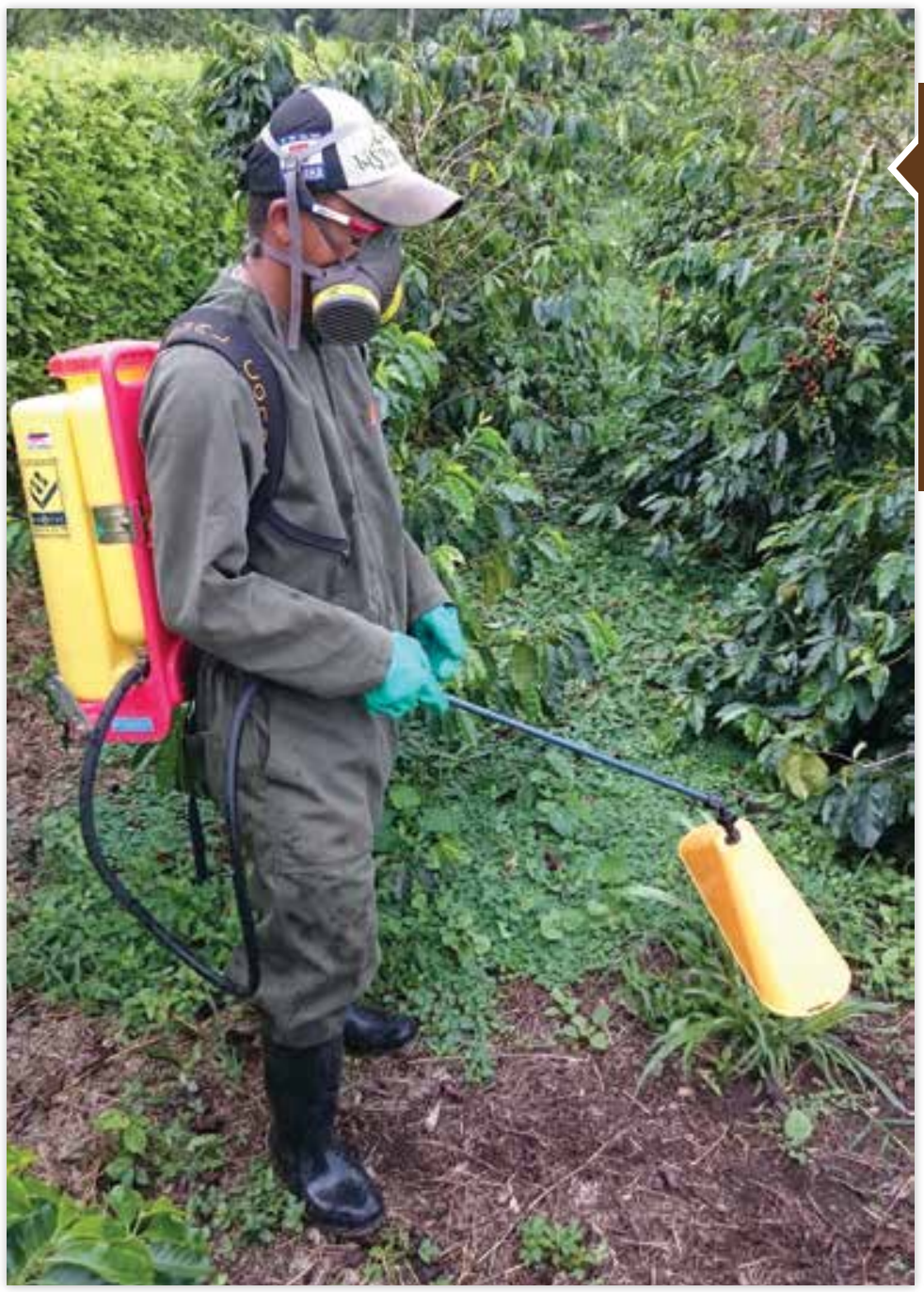

Operario realizando parcheo con equipo de aspersión y pantalla protectora. 
Manejo de arvenses en los cafetales en épocas secas.

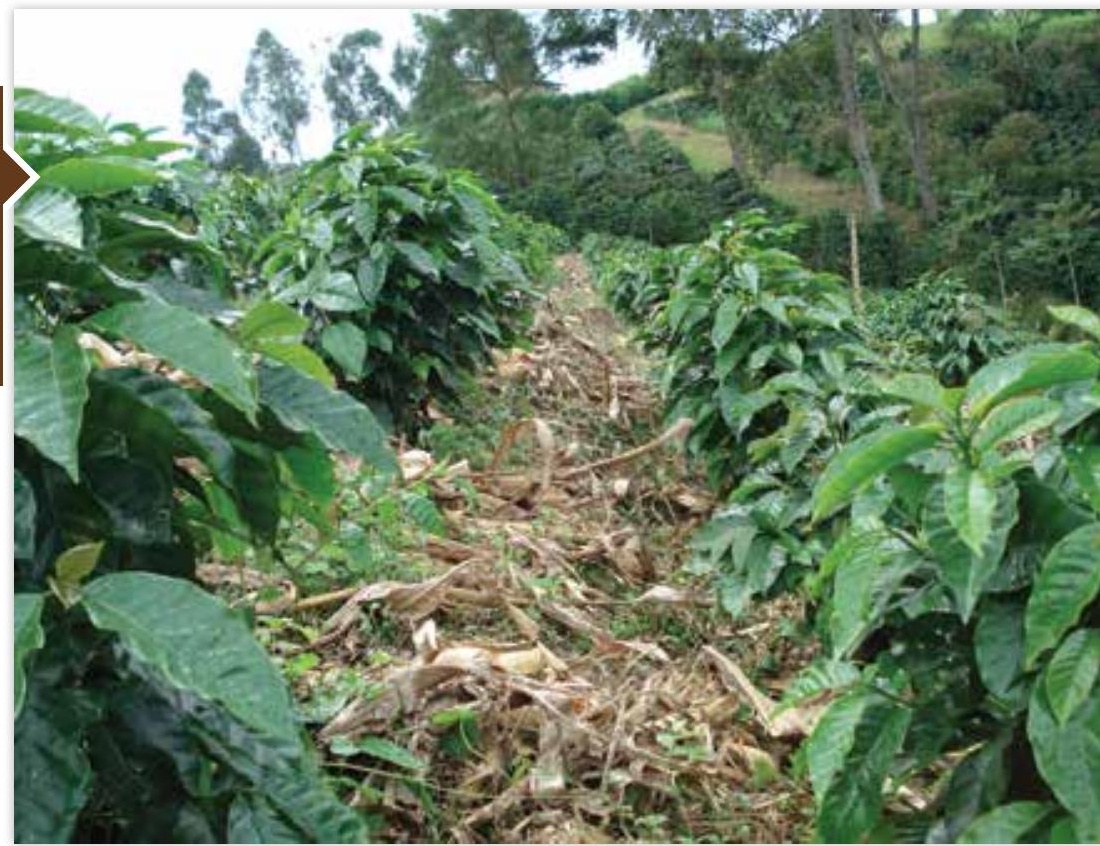

Tenga presente que al retornar las lluvias debe realizar las prácticas del MIA para continuar con el establecimiento de coberturas nobles. 


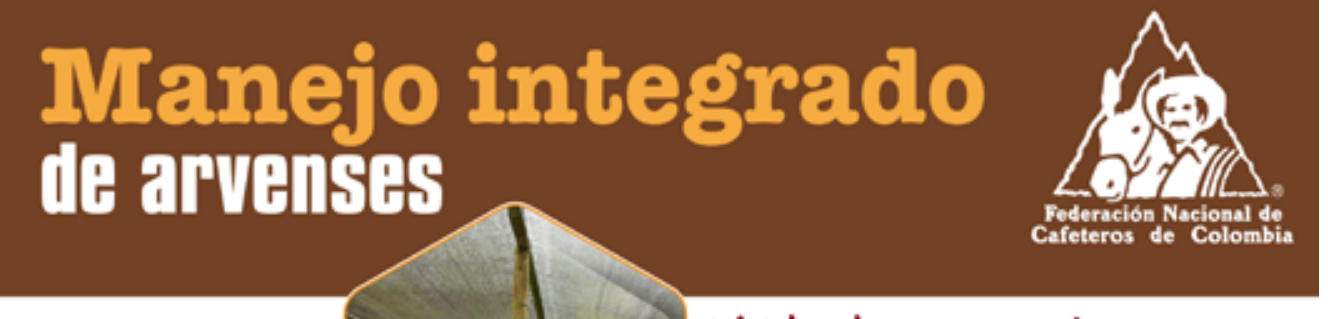

\section{Método manual}

Arranque las arvenses con la mano en el almácigo, en el plato del café y en las calles cuando haya venadillo o bejucos.

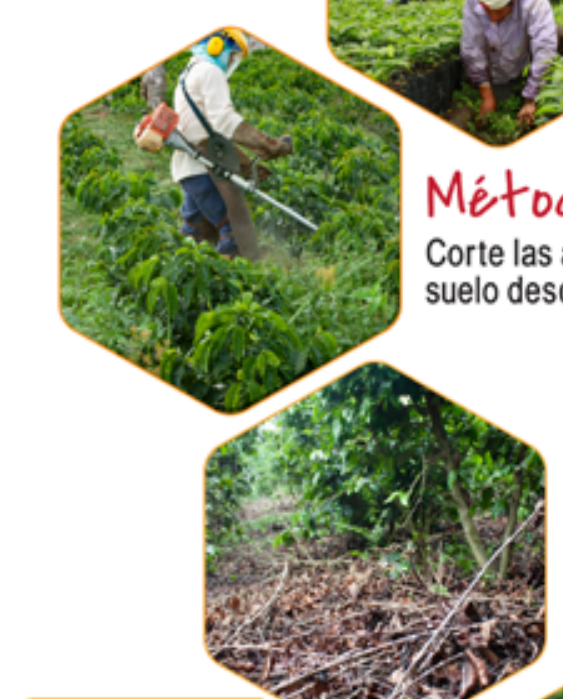

\section{Método cultural}

Realice prácticas Que favorezcan más al café Que a las arvenses; por ejemplo, la fertilización, distancias de siembra adecuadas, la cobertura del suelo con ramillas y hojarasca.

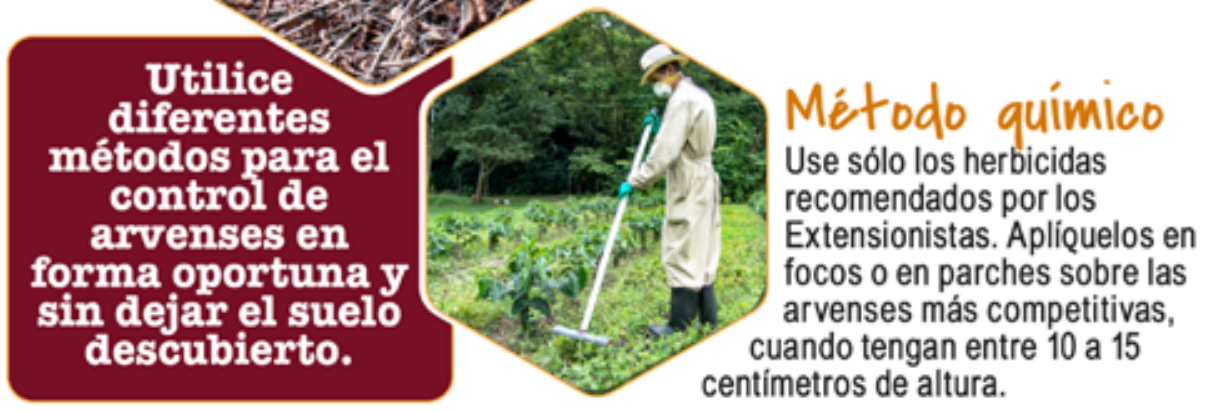

\section{Caficultor:}

El manejo integrado de arvenses mantiene la producción, reduce los costos de las desyerbas y previene la erosión del suelo.

\section{consulte con su Extensionista}

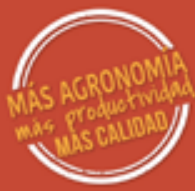

